\title{
Etude de la morbidité des enfants de 0 à 59 mois à la mutuelle DEMBA NYUMAN en Commune VI du district de Bamako
}

\section{Study of the morbidity of children from 0 to 59 months at the DEMBA NYUMAN mutual in Commune VI of Bamako}

Sangho $\mathrm{F}^{1,2,3^{*}}$, Coulibaly $\mathrm{CA}^{2}$, Diarra $\mathrm{O}^{4}$, Sangho $\mathrm{A}^{5}$, Sangho $\mathrm{O}^{1,2,6}$, Sangho $\mathrm{F}^{7}$, Touré $\mathrm{F}^{8}$, Telly $\mathrm{N}^{2,9}$, Diallo $\mathrm{B}^{10}$, Diarra $\mathrm{MM}^{11}$, Togo $\mathrm{Y}^{1}$, Oumar $\mathrm{YA}^{6}$, Coulibaly $\mathrm{M}^{6}$, Somboro $\mathrm{AH}^{6}$, Dackouo $\mathrm{T}^{6}$, Konaté $\mathrm{A}^{6}$, Sawadogo $\mathrm{S}^{6}$, Sissoko N6, Kéïta ${ }^{6}$, Camara B6 , Dao O13, Dao $\mathrm{S}^{14}$, Sangho H²

1. Département d'Enseignement et de Recherche des Sciences Biologiques et Médicales, Faculté de Pharmacie (FAPH) / Université des Sciences, des Techniques et des Technologies de Bamako (USTTB), Mali

2. Département d'Enseignement et de Recherche en Santé Publique et Spécialités (DERSP), FMOS/USTTB, Mali

3. Direction de la Pharmacie et du Médicament (DPM), Bamako, Mali

4. Mutuelle DEMBA NYUMAN, Commune VI du district de Bamako

5. Département d'Enseignement et de Recherche des Sciences Pharmaceutiques, FAPH/USTTB, Mali

6. Agence Nationale de Télésanté et d'Informatique Médicale, Hamdallaye $\mathrm{ACl}$, Bamako, Mali

7. Cellule de Planification et de Statistique, Secteur Santé, Développement Social et Promotion de la Famille (CPS/SS-DSPF), Bamako, Mali

8. Programme National de lutte contre le Paludisme (PNLP), Bamako, Mali

9. Cellule Sectorielle de Lutte Contre le SIDA la Tuberculose et les Hépatites Virales (CSLS-TBH), Bamako, Mali

10. Centre Hospitalier Universitaire Odonto-Stomatologie (CHUCNOS), Bamako, Mali

11. Centre de Santé de Référence (CSRéf) de Kéniéba, Kayes, Mali

12. Marie Stopes International, Mali

13. Service des Maladies Infectieuses et Tropicales, CHU Point G, Bamako, Mali

*Auteur correspondante : Dr Fanta Sangho, Maître Assistante Santé Communautaire au DER des Sciences Biologiques et Médicales, FAPH/USTTB/Mali, (00223) 667827 28, sanghofanta@gmail.com

\section{Résumé :}

Introduction : L'objectif de cette étude était d'étudier la morbidité des enfants de 0 à 59 mois à la Mutuelle DEMBA NYUMAN en commune VI du district de Bamako. Matériel et méthodes : II s'agissait d'une étude transversale avec recueil des données prospectives allant du 1 er Janvier au 31 Décembre 2018. Nous avons recensé 430 patients répondant aux critères d'inclusion. Résultats : Les enfants âgés de 12 à 23 mois étaient les plus représentés soit $23,7 \%$ avec une prépondérance féminine $(50,2 \%)$, la majorité habitait à Niamakoro (76\%). La diarrhée associée aux vomissements constituait le motif de consultation le plus fréquent $(22,1 \%)$, suivi de la rhinorrhée accompagnée de la toux (18,8\%). Le Test de diagnostic (TDR) du paludisme était l'examen complémentaire le plus demandé soit $58,50 \%$. Les principales pathologies responsables de morbidité étaient : le paludisme (34,88\%), les infections respiratoires hautes $(14,65 \%)$, et les infections respiratoires basses $(13,26 \%)$. Le schéma thérapeutique utilisé, parmi les enquêtés atteints de paludisme, $31,33 \%$ ont été traité par principalement atrhemeter+antibiotique+antipyrétique ; et ceux atteints de gastroentérite par du métronidazole + Cotrimoxazole + SRO (35,19\%). Conclusion : la morbidité chez les enfants de 0 à 59 mois était dominée par deux des quatre morbidités les plus fréquentes chez les enfants de cette tranche d'âge ; à savoir le paludisme suivi des infections respiratoires.

Mots clés : Mutuelle DEMBA NYUMAN, enfants de 0 à 59 mois, morbidité

Abstract:

Introduction: The objective of this study was to determine the morbidities of children aged 0 to 59 months at the DEMBA NYUMAN Mutual in commune $\mathrm{VI}$ of Bamako. Material and methods: This was a cross-sectional study with collection of prospective data from January 1 to December 31, 2018. We identified 430 patients meeting the inclusion criteria. Results: Children aged 12 to 23 months were the most represented, $23.7 \%$ with a preponderance of women $(50.2 \%)$, the majority lived in Niamakoro (76\%). Diarrhea associated with vomiting was the most frequent reason for consultation (22.1\%), followed by rhinorrhea with cough (18.8\%). The diagnostic test (RDT) for malaria was the most requested additional test at $58.50 \%$. The main pathologies responsible for morbidity were: malaria $(34.88 \%)$, upper respiratory infections (14.65\%), and lower respiratory infections $(13.26 \%)$. The therapeutic schema used, among the respondents suffering from malaria, $31.33 \%$ were treated with mainly arthemeter + antibiotic + antipyretic; and those with gastroenteritis with metronidazole + Cotrimoxazole + ORS (35.19\%). Conclusion: Morbidity in children aged 0 to 59 months was dominated by two of the four most frequent morbidity in children of this age group, namely malaria followed by respiratory infections.

Keywords: DEMBA NYUMAN mutual, children aged 0 to 59 months, morbidities

\section{Introduction}

La morbidité désigne l'exposition d'une population à des affections qui ne sont pas nécessairement mortelles. C'est la fréquence avec laquelle une population est atteinte par une maladie, une défectuosité physique, un traumatisme (1). La mortalité quant à elle se définit comme l'action de la mort sur une population exposée en un lieu et dans un espace de temps déterminé (1). La morbidité et la mortalité des enfants de moins de 5 ans restent un problème majeur de santé publique dans le monde, surtout dans les pays en développement.

En 2017 selon l'OMS/UNICEF, le nombre d'enfants de moins de 5 ans décédés à travers le monde est passé 
respectivement de 93 décès pour 1000 naissances vivantes en 1990 à 39 décès pour 1000 naissances vivantes en 2017 parmi lesquels $50 \%$ des décès ont lieu en Afrique Subsaharienne et $30 \%$ en Asie du Sud Est.

En Afrique sub-saharienne un enfant sur 13 meurt avant son $5^{\mathrm{e}}$ anniversaire (2). Dans les pays à revenu élevé, ce chiffre chute à un enfant sur 185. La plupart de ces décès sont dues à des causes que l'on peut éviter ou traiter notamment les complications à la naissance, la diarrhée, le paludisme et la septicémie néonatale.

Au Mali, selon EDSM V, la morbidité chez les enfants de moins de 5 ans est dominée principalement par le paludisme, les infections respiratoires aigües (IRA), la diarrhée, la rougeole et la malnutrition (3). Une étude réalisée au Centre de santé communautaire( CSCom) de Bacodjicoroni en 2014 avait trouvé le paludisme dans $42,6 \%$, l'IRA dans $31,8 \%$ et la diarrhée dans $11,6 \%$ (4). A notre connaissance, aucune étude n'a été réalisée dans le centre DEMBA NYUMAN malgré le taux de morbidité élevé au Mali, c'est ce qui a motivé le choix de ce thème. L'Objectif était d'étudier la morbidité des enfants de 0 à 59 mois à la mutuelle DEMBA NYUMAN en commune VI du district de Bamako.

\section{Matériel et méthodes}

1. Cadre d'étude :

L'étude a été réalisée au centre de santé mutuelle DEMBA NYUMAN en Commune VI du district de Bamako.

1.1. Présentation de la mutuelle DEMBA NYUMAN (1): II est créé une mutuelle dénommée DEMBA NYUMAN. Elle est établie à Bamako. Elle est régie par la loi 96-022 du 21 février 1996 régissant la mutualité en république du Mali, le dossier de la mutuelle enregistré sous le numéro 3014 du 6 mai 2009 (1) . Les objectifs se résument ainsi qu'il suit :

$>$ Assurer la prise en charge partielle des dépenses de santé de ses membres et de leur famille ;

$>$ Contribuer à la protection de l'état de santé de ses membres et de leur famille ;

$>$ Assister les adhérents lors des évènements sociaux ;

$>$ Contribuer à l'amélioration des conditions de vie de ses adhérant et leur famille.

Situé à environ 400 mètres de l'ancien marché de Niamakoro côté Sud, de par ses activités de qualités rendues le centre donne de grandes satisfactions à la population de Niamakoro et ainsi que d'autres populations riveraines du quartier. Les activités du centre sont entre autres : les consultations médicales, les soins infirmiers, la vaccination, les accouchements, les consultations pré natales (CPN) et post natales (CPoN), les examens de laboratoire (GE, test d'Emmel, sérologie toxoplasmose, groupage rhésus, glycémie, ASLO, sérologie Bordet Wassermann, albumine sucre, test de grossesse, sérologie et beta $\mathrm{HCG}$ ) ;

$>$ Autres examens complémentaires échographie (mardi et vendredi)
Le centre est un bâtiment d'un étage :

Au rez-de-chaussée : le guichet, la salle d'urgence, la pharmacie, la salle de CPN, le magasin pour archivage, le dortoir gardien, la salle d'accouchement, le magasin de stockage, les toilettes patient et personnel.

À l'étage : on a deux blocs

Le bloc A : le bureau du DTC, la salle de soins, la salle de perfusion, la salle d'attente, la direction technique de la mutuelle, la salle de garde.

Le bloc B : le bureau du médecin, la salle d'attente, la salle soin, le laboratoire, le bureau du gestionnaire comptable, la salle de perfusion.

\subsection{Personnel du centre}

Le centre est composé de personnels suivants : un médecin, deux infirmiers d'Etat, deux sages-femmes, un gérant de pharmacie, deux techniciennes laboratoires, trois infirmières obstétriciennes, une matrone, deux comptables, un gestionnaire et deux gardiens

\section{Type et période d'étude}

II s'agissait d'une étude transversale avec recueil des données du 01 Janvier au 31 Décembre 2018.

\section{Population d'étude}

L'étude a concerné les enfants de 0 à 59 mois qui ont été enregistrés dans les registres de consultations curatives de la mutuelle DEMBA NYUMAN.

\section{Critères d'inclusion}

Ont été inclus dans notre étude les enfants de 0 à 59 mois, qui ont été enregistrés dans les registres de consultation du DEMBA NYUMAN en 2018 et dont les informations sont complètes

\section{Critères de non inclusion}

N'ont pas été inclus dans notre étude les enfants de plus de 59 mois, dont les informations sont incomplètes.

\section{Echantillonnage}

L'étude a porté sur 430 enfants ayant consulté pendant la période d'étude

\section{Techniques de collecte des données}

Les données ont été collectées à l'aide d'une fiche de dépouillement. Le support a été les registres de consultation de 2018.

\section{Variables}

Sexe, Age, Résidence, Motif de consultation, Examens complémentaires : ils sont réalisés au laboratoire du centre par un laborantin, Résultats de l'examen demandé, Diagnostic, Traitement, Référence, Motifs de référence.

\section{Traitement et analyse des données}

Les données ont été saisies, traitées et analysées sur le logiciel SPSS 22.0 et Excel 2016. Les données ont été présentées sous forme tabulaire. Les effectifs et leurs proportions ont été présentés

\section{Aspects éthiques}

Les données clinique et biologique ont fait l'objet d'une stricte confidentialité et l'anonymat des patients a été préservé, par l'attribution de numéros à toute des fiches d'enquête. 


\section{Résultats}

Durant notre période d'étude, nous avons colligés 430 enfants âgés de 0 à 59 mois à la mutuelle Demba Nyuman en commune VI du district de Bamako.

1. Caractéristiques sociodémographiques (tableau I). Parmi les enquêtés, 23,7\% étaient majoritairement âgés de 12-23 mois, suivis de la tranche d'âge de 48-59 mois dans $20,9 \%$ des cas. Le sexe féminin prédominait dans $50,2 \%$ des cas. La majorité des enquêtés soit $76 \%$ résidaient à Niamakoro. Parmi les enquêtés les bambaras étaient majoritairement représentés avec $39,30 \%$.

\section{Morbidité}

\section{-Motifs de consultation}

La diarrhée + vomissement suivi de la rhinorrhée+ toux dominaient avec respectivement un taux de $22,1 \%$ et $18,8 \%$.

\section{-Examens complémentaires}

Au total $68,37 \%$ des enquêtés avaient fait les examens complémentaires demandés

TDR était l'examen le plus demandé soit $58,50 \%$, suivi de la GE +TDR dans $30 \%$ des cas. Parmi les enquêtés, $36,1 \%$ avaient un TDR négatif ; $22,4 \%$ avaient un TDR positif, $1,4 \%$ avaient un Widal positif et $1 \%$ avaient une GE positive, TDR positif, Widal positif (tableau II).

Le paludisme était la pathologie principalement rencontrée dans $34,88 \%$ suivi de l'IRA haute dans $14,65 \% ; 12,56 \%$ avaient une gastroentérite ; 7,20\% avaient une infection cutanée ; $1,62 \%$ avaient de la fièvre typhoïde. Sur les autres diagnostics, il y avait principalement $13,23 \%$ cas d'accident domestique suivi de $11,76 \%$ cas d'otite moyenne aigue (tableau III).

Le paludisme était la pathologie fréquemment rencontrée. Parmi les pathologies associées au paludisme, l'IRA basse était la plus retrouvée avec $38,66 \%$, suivi de la pharyngite dans $20 \%$ et de la fièvre typhoïde dans $2 \%$ des cas (tableau IV).

\section{Prise en charge thérapeutique}

Parmi les enquêtés atteints de paludisme, $31,33 \%$ avaient été traités par Arthemeter, un Antipyrétique et un Antibiotique en association, suivi de l'artesunate, antipyrétique et antibiotique dans $24,66 \%$ de cas (tableau V).

Parmi les enquêtés atteints d'IRA haute, $38,10 \%$ avaient été traités majoritairement par un Antibiotique associé à un antipyrétique (tableau VI).

Parmi les enquêtés atteints d'IRA basse, $29,82 \%$ avaient reçu un antipyrétique associé à un antibiotique principalement, suivi de Apdyl+Antipyrétique+ Antibiotique dans $17,54 \%$ des cas (tableau VI).

Parmi les enquêtés atteints de diarrhée, 35,19\% avaient reçu le Métronidazole, Cotrimoxazole et le SRO majoritairement, suivi de Métronidazole seul dans 16,67\% des cas (tableau VII).

\section{DISCUSSION}

Notre étude s'est déroulée à la mutuelle DEMBA NUYMAN en commune VI de district de Bamako. Les limites rencontrées lors de notre étude sont : les dossiers retirés pour informations manquantes.

\section{A. Caractéristiques sociodémographiques $\checkmark$ Age}

Parmi les enquêtés, la tranche d'âge de 12-23 mois était la plus représentée avec $23,7 \%$. Ceci pourrait s'expliquer par la baisse d'anticorps maternel à cette période et le développement progressif de l'immunité propre des enfants. Notre résultat est similaire à celui de Keita qui a trouvé la même tranche d'âge avec 26,2\% (4)

\section{$\checkmark$ Sexe}

Dans notre étude on notait une prédominance du sexe féminin $(50,2 \%)$. Ce résultat diffère de celui de Kéita (4) et Traoré B (5) qui avaient trouvé une prédominance masculine soit respectivement $52,4 \%$ et $52,8 \%$. Ce résultat discordant pourrait s'expliquer par la faible taille de leur échantillon par rapport à la nôtre.

\section{$\checkmark$ Résidence}

La majorité des enquêtés résidaient à Niamakoro soit $76 \%$. Ce qui pourait s'expliquer par l'accès géographique du fait que le centre de santé se trouve dans ce quartier.

\section{B. Morbidité}

$\checkmark$ Les motifs de consultation

Dans notre étude, la diarrhée associée au vomissement représentait le principal motif de consultation, soit $22,1 \%$. Selon EDSM VI, $17 \%$ des enfants ont souffert de diarrhée au cours des deux dernières semaines avant l'enquête (6). Notre résultat diffère de ceux de Keita (4) et Diarra A (7) qui retrouvaient majoritairement la fièvre avec 32,5 et $35,8 \%$, alors qu'elle ne représentait que $14,7 \%$ dans notre série. Ceci pourrait s'expliquer par le fait que les parents ont pour habitude de donner devant toute fièvre du traitement à base d'antipyrétique avant de consulter.

\section{$\checkmark$ Examens complémentaires}

Dans notre étude $68,37 \%$ avaient fait des examens complémentaires, dont le TDR fut l'examen le plus demandé à $58,50 \%$. Nos données diffèrent de ceux de Diarra A (7) qui avait trouvé que $14 \%$ avaient les examens complémentaires, dont la goutte épaisse représentait $50 \%$.

\section{Diagnostics : \\ $\checkmark$ Paludisme}

Le paludisme était la pathologie dominante avec une fréquence de $34,88 \%$. Ce résultat est inférieur à celui de Traoré B (5), de Doumbia AK et al. (8) et de Keita (4) chez lesquels le paludisme représentait la première cause de morbidité avec respectivement $50,5 \%, 45 \%$ et $42,6 \%$. Au Mali le paludisme est classé par les statistiques sanitaires comme la première cause de mortalité et morbidité infantiles (9). Dans notre étude il y a $25,33 \%$ de paludisme chez les enfants de $48-59$ mois et $4 \%$ chez les moins de 6 mois. La présence du paludisme confirmé chez les enfants de moins de 6 mois pourrait s'expliquer par l'absence de prise du traitement préventif du paludisme (sulfadoxine-pyriméthamine) pendant la grossesse, le non suivi de la grossesse et l'insuffisance de l'allaitement 
maternel. II est à noter tout de même que la prévention du paludisme dans certaines conditions n'apparait pas comme facteur protecteur des enfants et ne réduit pas l'incidence de la maladie (10). Par ailleurs, l'allaitement maternel aiderait les enfants à la réduction de leurs morbidités, comme par exemple dans l'étude de Houle B et al. qui trouve qu'il protège contre l'obésité (11).

Parmi les pathologies associées au paludisme, IRA basse était la plus retrouvée avec $38,66 \%$; suivi de la pharyngite dans $20 \%$ et de la fièvre typhoïde dans $2 \%$ des cas.

\section{$\checkmark$ Les IRA}

Dans notre étude les IRA venaient en second lieu après le paludisme avec un taux de 27,91\%. Par contre, selon EDS-RDCII (2013-2014) (12), I'IRA représentait la seconde pathologie avec $7 \%$ chez les enfants de moins de 5 ans.

Notre résultat est inférieur à celui rapporté par Keita (4) qui trouve $31,8 \%$. Les IRA constituent aussi un fardeau dans la morbi-mortalité des enfants. C'est pourquoi, il avait été recommandé par Bénet et al. d'instituer une vaccination pour sa prévention (13). Jefferson et al. mettent aussi l'accent sur la vaccination pour la prévention de la grippe (14). D'autres études ont mis en évidence les fardeaux des IRA à travers des revues systématiques et la modélisation (15-17).

\section{$\checkmark \quad$ La gastroentérite}

La gastro-entérite a constitué la troisième pathologie rencontrée avec une fréquence de $12,56 \%$. Keita (4) dans son étude avait également trouvé la diarrhée comme troisième pathologie avec $11,6 \%$. L'EDSM VI a trouvé que la prévalence de la diarrhée est particulièrement élevée chez les enfants de $48-59$ mois $(54,9 \%)$ (6). Cette pathologie est souvent citée comme première cause de mortalité des enfants et conduit les parents à des recours thérapeutiques autant traditionnels $(57 \%)$ que médical (27\%) (18).

\section{Prise en charge thérapeutique}

Dans notre étude, $34,88 \%$ des enquêtés atteints de paludisme avaient été traités par les CTA à $44,19 \%$ et $41,86 \%$ par de l'arthemeter injectable. Le traitement utilisé pour traiter le paludisme associé aux autres pathologies était majoritairement artemether+antipyrétique+antibiotique dans 31,33\%, suivi de l'artesun+antipyrétique+antibiotique dans $24,66 \%$ de cas.

Par rapport au traitement de l'infection respiratoire haute aiguë, $14,65 \%$ des enquêtés atteints d'IRA haute avaient été traités par un antibiotique+antipyrétique à un taux de $38,10 \%$ suivi de litacold+antibiotique dans $22,22 \%$ des cas.

En ce qui concerne le traitement de l'infection respiratoire basse, $13,26 \%$ des enquêtés atteints d'IRA basse avaient été traités par antipyrétique+antibiotique à un taux de 29,82\% ; majoritairement suivi de Apdyl+Antipyrétique+ Antibiotique dans $17,54 \%$ des cas.

Concernant les enquêtés atteints de gastroentérite, $12,56 \%$ avaient été traités par le metronidazole+cotrimoxazole+SRO à un taux de $35,19 \%$ suivis de métronidazole seul dans $16,67 \%$ des cas. L'utilisation de SRO, dans le traitement des enquêtés atteints de diarrhée, pourrait être expliquée par les pertes électrolytiques qu'engendre la diarrhée.

L'EDSM V a montré que plus du tiers (37\%) avaient reçu des sachets de SRO, 18\% des enfants avaient reçu une solution d'eau, de sel et de sucre préparée à la maison. L'EDS-RDCII (2013-204) avait trouvé que 39\% des enfants malades de diarrhée avaient reçu une solution de SRO (12). II est important de noter que l'initiation précoce des traitements est un élément déterminant la réduction de l'état morbide de même que la mortalité (19). C'est pourquoi certains auteurs mettent l'accent sur le rôle des parents, après leur formation ou le renforcement de leur sensibilisation, dans la prise en charge des enfants $(20,21)$. Ce rôle pourra être renforcé par des stratégies supplémentaires comme le suggère Winch $P$ et al. dans son étude sur l'introduction du zinc dans la prise en charge de la diarrhée (22).

\section{CONCLUSION}

Au terme de notre étude, les enquêtés âgés de 12 à 23 mois étaient les plus représentés avec une prépondérance féminine, la majorité habitait à Niamakoro. La diarrhée associée aux vomissements constituait le motif de consultation le plus fréquent, suivi de la rhinorrhée accompagnée de la toux. Le Test de diagnostic (TDR) du paludisme était l'examen complémentaire le plus demandé. Les principales pathologies responsables de morbidité étaient : le paludisme, les infestions respiratoires hautes et basses. Parmi les enquêtés atteints de paludisme, les CTA étaient majoritairement prescrits ; et ceux atteints de gastroentérite par du métronidazole + Cotrimoxazole + SRO

\section{Références}

1. Ministère de la Santé de la Solidarité et des Personnes Agées (MSSPA) Système National d'Information Sanitaire (SNIS) 2005 ; Bilan des activités 2004-2005.

2. UNICEF, WHO, WORD Bank UPD.

levels and frends in child mortality report 2018 Estimates developed by the UN Inter-agency Group for Child Mortality Estimation. 2018. 48 p.

3. EDSM V Cellule de planification et de statistique (CPS/SSDSPF). Institut national de la statistique ( INSTAT/MPATP), INFO-STAT et ICF international. Enquete démographique et de santé au Mali (EDSM V) 2012-2013.Rockville, Maryland, USA: CPS, INSTAT, INFO-S.

4. SACKO MMKK épouse. Etude de la morbidité chez les enfants de 0 à 59 mois au centre de san-té communautaire de baco djicoroni en commune $\mathrm{V}$ du district de Ba-mako. 
5. B T. Etude de la morbidité des enfants de 0 à 59 mois au centre de santé communautaire de Bacodjicoroni de 2002 à 2009.

6. Institut National de la Statistique (INSTAT), Cellule de Planification et de Statistique Secteur Santé-Développement Social et Promotion de la Famille (CPS/SS-DS-PF), ICF. Sixième Enquête Démographique et de Santé au Mali 2018. Indicateurs Clés. [Internet]. Bamako, Mali, et Rockville, Maryland, USA: INSTAT, CPS/SS-DSPF, et ICF; 2019 [cité 21 mai 2019]. Disponible sur:

https://dhsprogram.com/pubs/pdf/PR115/PR115.pdf 7. A. D. Etude de la mortalité et morbidité infanto-juvénile au centre de santé de référence de Niono de 2000 à 2004. Thèse. Médecine. Année 2005-2006.Bamako. 05-M-228. n 144.

8. Doumbia AK, Togo B, Togo P, Traore F, Coulibaly O, Dembele A, et al. MORBIDITE ET MORTALITE CHEZ LES ENFANTS DE 01 A 59 MOIS HOSPITALISES AU SERVICE DE PEDIATRIE GENERALE DU CHU GABRIEL TOURE DE JANVIER A DECEMBRE 2013. Rev Malienne D'Infectiologie Microbiol. 17 nov 2016;8:54-62.

9. Santos D. Manuel de nutrition africaine. Paris : Karthala, 1987, 470p.

10. Dicko A, Barry A, Dicko M, Diallo AI, Tembine I, Dicko Y, et al. Malaria Morbidity in Children in the Year after They Had Received Intermittent Preventive Treatment of Malaria in Mali: A Randomized Control Trial. PLoS ONE [Internet]. 12 août 2011 [cité 16 janv 2021];6(8). Disponible sur:

https://www.ncbi.nlm.nih.gov/pmc/articles/PMC31 55530/

11. Houle B, Rochat TJ, Newell M-L, Stein A, Bland RM. Breastfeeding, HIV exposure, childhood obesity, and prehypertension: A South African cohort study. PLoS Med [Internet]. 27 août 2019 [cité 16 janv 2021];16(8). Disponible sur: https://www.ncbi.nlm.nih.gov/pmc/articles/PMC67 11496/

12. Ministère de la santé: Enquete deémographique de la santé de la République démocratique du Congo II, 2013-2014. page 2. 13. Bénet T, Sylla M, Messaoudi M, Sánchez Picot V, Telles J-N, Diakite A-A, et al. Etiology and Factors Associated with Pneumonia in Children under 5 Years of Age in Mali: A Prospective CaseControl Study. PLoS ONE [Internet]. 22 déc 2015 [cité 16 janv 2021];10(12). Disponible sur: https://www.ncbi.nlm.nih.gov/pmc/articles/PMC46 87909/

14. Jefferson T, Rivetti A, Di Pietrantonj C, Demicheli V. Vaccines for preventing influenza in healthy children. Cochrane Database Syst Rev [Internet]. 1 févr 2018 [cité 16 janv 2021];2018(2). Disponible sur:

https://www.ncbi.nlm.nih.gov/pmc/articles/PMC64 91174/

15. Wang X, Li Y, O'Brien KL, Madhi SA, Widdowson M-A, Byass P, et al. Global burden of respiratory infections associated with seasonal influenza in children under 5 years in 2018: a systematic review and modelling study. Lancet Glob Health. 20 févr 2020;8(4):e497-510.

16. Wang X, Li Y, Deloria-Knoll M, Madhi SA, Cohen C, Ali A, et al. Global burden of acute lower respiratory infection associated with human metapneumovirus in children under 5 years in 2018: a systematic review and modelling study. Lancet Glob Health. 26 nov 2020;9(1):e33-43. 17. Zhang S, Sammon PM, King I, Andrade AL, Toscano CM, Araujo SN, et al. Cost of management of severe pneumonia in young children: systematic analysis. J Glob Health [Internet]. [cité 16 janv 2021];6(1). Disponible sur: https://www.ncbi.nlm.nih.gov/pmc/articles/PMC48 71066/

18. Farag TH, Kotloff KL, Levine MM, Onwuchekwa U, Van Eijk AM, Doh S, et al. Seeking Care for Pediatric Diarrheal Illness from Traditional Healers in Bamako, Mali. Am J Trop Med Hyg. 10 juill 2013;89(1 Suppl):21-8.

19. Johnson AD, Thomson DR, Atwood S, Alley I, Beckerman JL, Koné I, et al. Assessing Early Access to Care and Child Survival during a Health System Strengthening Intervention in Mali: A Repeated Cross Sectional Survey. PLoS ONE [Internet]. 11 déc 2013 [cité 16 janv 2021];8(12). Disponible sur:

https://www.ncbi.nlm.nih.gov/pmc/articles/PMC38 59507/

20. Barlow J, Bergman H, Kornør H, Wei Y, Bennett C. Group-based parent training programmes for improving emotional and behavioural adjustment in young children. Cochrane Database Syst Rev [Internet]. 1 août 2016 [cité 16 janv 2021];2016(8). Disponible sur: https://www.ncbi.nlm.nih.gov/pmc/articles/PMC67 97064/ 
21. Sangho H, Keïta AS, Keita HD, Belemou B, Keïta FD, Bamba SI, et al. Formation des mères : stratégie pour améliorer le traitement des infections respiratoires aiguës au Mali. Sante Publique

(Bucur). 8 juin 2012;Vol. 24(HS):23-31.

22. Winch PJ, Gilroy KE, Doumbia $S$,

Patterson AE, Daou Z, Diawara A, et al.
Operational Issues and Trends Associated with the Pilot Introduction of Zinc for Childhood Diarrhoea in Bougouni District, Mali. J Health Popul Nutr. juin 2008;26(2):151-63.

Liste des tableaux :

Tableau I : Répartition des enquêtés selon les caractéristiques sociodémographiques, $\mathrm{N}=430$

\begin{tabular}{|c|c|c|}
\hline Caractéristiques & Effectif & $\%$ \\
\hline \multicolumn{3}{|l|}{ Age (en mois) } \\
\hline$<6$ mois & 58 & 13,49 \\
\hline $6-11$ & 70 & 16,28 \\
\hline $12-23$ & 102 & 23,72 \\
\hline $24-35$ & 63 & 14,65 \\
\hline $36-47$ & 47 & 10,93 \\
\hline $48-59$ & 90 & 20,93 \\
\hline \multicolumn{3}{|l|}{ Sexe } \\
\hline masculin & 214 & 49,77 \\
\hline féminin & 216 & 50,23 \\
\hline \multicolumn{3}{|l|}{ Résidence } \\
\hline Niamakoro & 327 & 76,05 \\
\hline Kalaban coura & 38 & 8,84 \\
\hline Hors commune VI & 25 & 5,81 \\
\hline Faladiè & 14 & 3,26 \\
\hline Sokorodji & 8 & 1,86 \\
\hline Yirimadjo & 8 & 1,86 \\
\hline Banankabougou & 7 & 1,63 \\
\hline Sabalibougou & 2 & 0,47 \\
\hline Moussabougou & 1 & 0,23 \\
\hline \multicolumn{3}{|l|}{ Ethnie } \\
\hline Bambara & 169 & 39,30 \\
\hline Soninké & 82 & 19,07 \\
\hline Peulh & 64 & 14,88 \\
\hline Malinké & 34 & 7,91 \\
\hline Sonrhaï & 20 & 4,65 \\
\hline Dogon & 21 & 4,88 \\
\hline Autres* & 13 & 3,02 \\
\hline Bozo & 10 & 2,33 \\
\hline Senoufo & 7 & 1,63 \\
\hline Mianka & 5 & 1,16 \\
\hline Mossi & 3 & 0,70 \\
\hline Bobo & 2 & 0,47 \\
\hline
\end{tabular}

Tableau II : Répartition des enquêtés selon le type d'examens complémentaires demandés et de son résultat, N=294

\begin{tabular}{llll}
\hline \multicolumn{1}{l}{ Type d'examen demandé } & Résultat des examens & $\mathrm{n}(\%)$ \\
\hline TDR & $172(58,50)$ & TDR négatif & $106(36,05)$ \\
& & TDR positif & $66(22,45)$ \\
\hline GE + TDR & \multirow{2}{*}{$88(29,93)$} & GE positive +TDR positif & $44(14,97)$ \\
& & GE positive + TDR négatif & $28(9,52)$ \\
& & GE négative + TDR négatif & $16(5,44)$ \\
\hline GE & $27(9,18)$ & GE positive & $15(5,10)$ \\
& & GE négative & $12(4,08)$ \\
\hline GE + TDR + WIDAL & $7(2,38)$ & WIDAL positif & $4(1,36)$ \\
& & GE positive+ TDRpositif+ WIDALpositif & $3(1,02)$ \\
\hline
\end{tabular}


Tableau III : Répartition des enquêtés selon le diagnostic

\begin{tabular}{lcc}
\hline Diagnostic & Effectif & $\%$ \\
\hline Paludisme & 150 & 34,88 \\
IRA haute & 63 & 14,65 \\
IRA basse & 57 & 13,26 \\
Gastroentérite & 54 & 12,56 \\
Infection cutanée & 31 & 7,20 \\
Fièvre typhoïde & 7 & 1,62 \\
Accident domestique & 9 & 2,09 \\
Otite moyenne aigue & 8 & 11,76 \\
Brulure & 7 & 10,29 \\
Angine & 6 & 8,82 \\
Accident voie publique & 5 & 7,35 \\
Intoxication alimentaire & 4 & 5,88 \\
Candidose buccale & 4 & 5,88 \\
Otite purulente & 4 & 5,88 \\
Varicelle & 3 & 4,41 \\
Corps étrangers & 3 & 4,41 \\
Asthme & 2 & 2,94 \\
Furonculose & 2 & 2,94 \\
Conjonctivite & 2 & 2,94 \\
Pharyngite & 2 & 2,94 \\
Phimosis & 1 & 1,47 \\
Prurigo & 1 & 1,47 \\
Saignement ombilical & 1 & 1,47 \\
Fracture bras & 1 & 1,47 \\
\hline & & \\
\hline
\end{tabular}

Tableau IV : Répartition des enquêtés selon les principaux diagnostics en fonction de l'âge

\begin{tabular}{lccccccc}
\hline Age (mois) & \multicolumn{9}{c}{ Diagnostic } & Total \\
\cline { 2 - 6 } & Paludisme & $\begin{array}{c}\text { IRA } \\
\text { haute }\end{array}$ & IRA basse & $\begin{array}{c}\text { Fièvre } \\
\text { typhoïde }\end{array}$ & $\begin{array}{c}\text { Infection } \\
\text { cutanée }\end{array}$ & Autres \\
\hline$<6$ & 6 & 11 & 9 & 0 & 4 & 28 & 58 \\
$6-11$ & 26 & 9 & 9 & 0 & 5 & 21 & 70 \\
$12-23$ & 33 & 21 & 12 & 0 & 8 & 28 & 102 \\
$24-35$ & 27 & 6 & 8 & 1 & 4 & 17 & 63 \\
$36-47$ & 20 & 7 & 7 & 1 & 0 & 12 & 47 \\
$48-59$ & 38 & 9 & 8 & 5 & 9 & 21 & 90 \\
Total & 150 & 63 & 53 & 7 & 30 & 127 & 430 \\
\hline
\end{tabular}

Tableau V : Répartition des enquêtés atteints de paludisme selon le schéma thérapeutique de traitement ou protocole de traitement reçus

\begin{tabular}{lcc}
\hline Traitement & Effectif & $\%$ \\
\hline Arthemeter+ Antipyrétique+ Antibiotique & 47 & 31,33 \\
Artesunate+ Antipyrétique+ Antibiotique & 37 & 24,66 \\
CTA+ Anti pyrétique & 34 & 22,66 \\
CTA +Antipyrétique+ Antibiotique & 24 & 16,00 \\
Perfusion de quinine+ Antibiotique & 8 & 5,33 \\
Total & 107 & 100,00 \\
\hline
\end{tabular}


Tableau VI : Répartition des enquêtés atteints d'IRA haute selon les traitements reçus

\begin{tabular}{lcc}
\hline IRA haute & Effectif & $\%$ \\
\hline Antibiotique+ Antipyrétique & 24 & 38,10 \\
Antibiotique+ Litacold & 14 & 22,22 \\
Antibiotique+ Paidoterin & 10 & 15,87 \\
Antibiotique+ Antipyrétique+ & 8 & 12,70 \\
Prométhazine & & \\
Antibiotique+ Primalan & 3 & 4,76 \\
+Antibiotique+ Antipyrétique+ Paidoterin & 2 & 3,17 \\
Antipyrétique +Prométhazine & 2 & 3,17 \\
Total & 63 & 100,00 \\
\hline
\end{tabular}

Tableau VII : Répartition des enquêtés atteints d'IRA basse selon le regroupement des traitements reçus

\begin{tabular}{lcc}
\hline IRA Basse & Effectif & $\%$ \\
\hline Antipyrétique+ Antibiotique & 17 & 29,82 \\
Apdyl+Antipyrétique+ & 10 & 17,54 \\
Antibiotique & & 15,79 \\
Carbocysteine 2\%+Antibiotique & 9 & 10,53 \\
Dexamethasone + & 6 & \\
Antibiotique & 5 & 8,77 \\
Broncoxil+Antipyrétique + & 5 & 8,77 \\
Antibiotique & 5 & 8,77 \\
Carbocysteine 2\% & 5 & 100,00 \\
Broncoxil+Antibiotique & 57 & \\
Total & 57 & \\
\hline
\end{tabular}

Tableau VII : Répartition de enquêtés atteints de diarrhée selon regroupement de traitements reçus

\begin{tabular}{lcc}
\hline Traitement Diarrhée & Effectif & $\%$ \\
\hline Métronidazole & 9 & 16,67 \\
Métronidazole+cotrimoxazole+SRO & 19 & 35,19 \\
Métronidazole+cotrimoxazole+smecta & 6 & 11,11 \\
Métronidazole+SRO* & 7 & 12,96 \\
Métronidazole injectable+Ringer Lactate & 6 & 11,11 \\
Métronidazole +Antipyrétique injectable+SRO & 7 & 12,96 \\
Total & $\mathbf{5 4}$ & $\mathbf{1 0 0 , 0 0}$ \\
\hline
\end{tabular}

*SRO : solution de réhydratation orale 